\title{
PRÁCTICAS SOSTENIBLES Y SOCIALMENTE RESPONSABLES COMO MOTIVACIÓN PARA TRABAJAR EN EL SECTOR AGROALIMENTARIO GALLEGO
}

\section{María Garrido}

\section{Resumen}

El sector agroalimentario gallego está cada vez más implicado en que sus actividades empresariales comulguen con prácticas sostenibles y socialmente responsables. Cada vez las empresas son más conscientes de los problemas ambientales que afectan a nuestro planeta y se involucran más en ellos. Esto también se ve reflejado en los intereses de los empleados, tanto reales como potenciales, quienes se sentirán más motivados si trabajan en organizaciones que comulgan con prácticas sostenibles y socialmente responsables. Por ello, planteamos el presente estudio, con el objetivo de conocer cómo influye la implementación de este tipo de prácticas en los estudiantes gallegos a la hora de buscar trabajo en empresas del sector agroalimentario. Realizamos una encuesta a 186 estudiantes del grado de Administración y Dirección de Empresas de la Universidad de Santiago de Compostela y descubrimos que consideran muy importante la práctica de este tipo de actividades, especialmente aquellas acciones que van dirigidas hacia los empleados.

Palabras clave: responsabilidad social corporativa, sector agroalimentario gallego, empleados potenciales

\section{Introducción y objetivos}

El sector agroalimentario gallego siempre ha jugado un papel fundamental en la economía de Galicia, siendo una de las actividades principales de la comunidad. Datos del año 2019 indican que representa el $12,1 \%$ del total que genera la economía gallega, y es la quinta actividad más importante a nivel nacional (Observatorio Cajamar, 2019).

Según un estudio realizado en la actualidad por el Instituto Galego de Estadística (IGE, 2019), se puede afirmar que en términos de empleo, la industria agroalimentaria genera en Galicia 103.521 puestos de trabajo, lo que supone un 9,3\% del trabajo total, y aporta un 6,9\% del Producto Interior Bruto (PIB) de la economía autonómica (4.126 millones de euros). Dentro de esta industria, destacan principalmente la "Agricultura, ganadería, caza y servicios relacionados con ellas" y la "Pesca" y la "Industria del procesamiento de conservación de pescados, crustáceos y moluscos" siendo las actividades que más valor y empleos generan.

Dicho esto, es evidente la importancia que este sector tiene en la comunidad gallega, que a su vez, está reflejando un cambio de intereses dentro del mundo empresarial. Cada vez la sociedad está más mentalizada con el cuidado del medioambiente, y se preocupa más por cuestiones ambientales, la procedencia y calidad de los alimentos... (López-Iglesias, 2019), por lo tanto, las empresas deberían de cuidar sus procesos productivos, ser socialmente responsables y realizar actividades en aras de conseguir sostenibilidad.

Teniendo en cuenta que la industria alimentaria es uno de los principales sectores productivos que mayor impacto tiene sobre el medioambiente, y que cada vez la sociedad está más mentalizada, El Clúster Alimentario de Galicia (Clusaga) define como uno de sus objetivos prioritarios "mejorar la productividad y sostenibilidad y avanzar hacia la fábrica del futuro" y para ello recoge una serie de acciones centrales dentro de su Plan Estratégico. Es fundamental que las empresas se mentalicen para cuidar el sector e intentar cumplir con los objetivos mencionados por CLUSAGA (Iribarren et al., 2010).

La Responsabilidad Social Corporativa (RSC) y la sostenibilidad no es algo que vaya a influir sólo en los trabajadores de la empresa, si no que se anticipa a todo esto y ya se habla de la relación que hay entre la RSC y los empleados potenciales (Klimkiewicz y Oltra, 2017; Skudiene y Auruskeviciene, 2012). Hay un enfoque de investigación que analiza lo que buscan las personas desempleadas a la hora de demandar un trabajo. Existe una corriente que sugiere que las personas que están buscando empleo mostrarán cierta tendencia hacia las empresas que comulgan con sus valores personales (Aguilera et al, 2007). Con la intención de seguir esta línea de investigación, planteamos el presente estudio, con el objetivo de conocer cómo influye la implementación de este tipo de prácticas en los estudiantes gallegos a la hora de buscar trabajo en empresas del sector agroalimentario.

Para ello, hemos realizado una encuesta a los estudiantes del grado de Administración y Dirección de Empresas con el fin de conocer sus intereses por las prácticas de RSC a la hora de buscar trabajo en el sector agroalimentario y hemos descubierto que, como futuros empleados, son sensibles a este tema, si bien es cierto que muestran más sensibilidad hacia las actividades dirigidas hacia los empleados y medioambiente. 
Con este trabajo pretendemos contribuir con la literatura existente y demostrar el efecto que produce en los empleados potenciales este tipo de actividades y cómo la RSC puede convertirse en una ventaja competitiva para la empresa. Además, los resultados obtenidos nos permitirán mostrarle a las empresas qué esperan de ellas sus futuros empleados.

\section{Metodología}

\subsection{La muestra}

La muestra está formada por estudiantes del grado de Administración y Dirección de Empresas de la Facultad de Ciencias Económicas y Empresariales de la Universidad de Santiago de Compostela. Para conocer sus intereses a la hora de buscar empleo, como futuros empleados, se les hizo llegar una encuesta vía email a 407 alumnos, de los cuales respondieron 195, lo que supone un ratio de respuesta del 48\%. De esas 195 encuestas se eliminaron nueve por estar incompletas. Por lo tanto, la muestra final está formada por 186 estudiantes pertenecientes a los cuatro cursos del grado.

A estos estudiantes se les ha realizado una encuesta basándonos en Turker (2009) y añadiendo alguna modificación, en la que tenían que suponer una situación de búsqueda de empleo y responder qué aspectos valorarían a la hora de aplicar en una empresa de este sector.

\subsection{Resultados}

Tras dividir las actividades de RSC en cuatro bloques: hacia los stakeholders, hacia los empleados, hacia los clientes y hacia el gobierno; observamos que para los estudiantes, los cuatro grupos son de su interés a la hora de buscar empleo en el sector agroalimentario. De media, los cuatro bloques están valorados como "Bastante importantes". Si bien es cierto, que al ámbito que más importancia le dan es al de las políticas que van dirigidas hacia los empleados, donde todos los items han sido valorados como "Muy importantes" por la mayoría de los alumnos.

Gráfico 2: Interés de los estudiantes hacia los diferentes items de la RSC

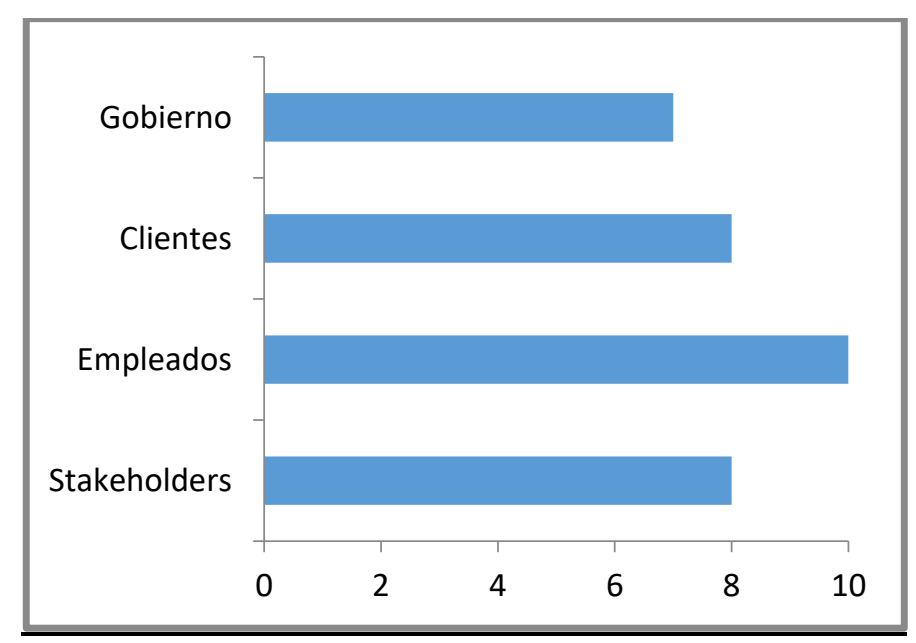

Fuente: elaboración propia

Si nos centramos en lo relativo a las acciones de RSC hacia los empleados, concretamente, lo que más interés ha despertado por parte de los alumnos, es que se preocupen por las necesidades y deseos de los empleados $(75,3 \%)$, seguido del hecho de que las organizaciones implementen políticas flexibles para brindar un buen equilibrio entre vida laboral y personal $(70,4 \%)$,

Llama la atención la neutralidad que expresan ante el hecho de que la empresa motive a sus empleados a participar en actividades de voluntariado, marcando un 41,4\% esta opción.

En el bloque de actividades hacia los stakeholders, tanto aquellas dirigidas hacia el mediambiente, como a mejorar la sociedad son calificadas como "bastante importantes", siendo el hecho de que apoye a las organizaciones no gubernamentales que trabajan en las áreas problemáticas la que menos interés presenta, con algunos alumnos que marcaron "ninguna" $(4,3 \%)$ y "un poco" $(8,6 \%)$. Dentro de este bloque, la que más alumnos han votado como "muy importante" es que la empresa en la que apliquen implemente programas especiales para minimizar su impacto negativo en el medioambiente $(36,6 \%)$. 
En el bloque de actividades hacia los clientes, que las empresas se preocupen por el grado de satisfacción de los clientes es la actividad que más interés despierta en los estudiantes, seguida de la información y de los requisitos legales.

Por último, en cuanto al bloque de acciones referentes a un buen hacer gubernamental, prácticamente un $15 \%$ de los encuestados muestra ningún interés, poco o neutral. En este caso, tanto el pago de impuestos como el cumplimiento con regulaciones legales están a la par.

Una vez comentado la importancia que los estudiantes le darían al hecho de que las empresas en las que buscan empleo realicen diferentes actividades de RSC, cabe destacar dos datos bastante reveladores que se han obtenido en la encuesta. Por una parte, llama la atención que el 13\% de los encuestados reconocen no saber qué significa el término Responsabilidad Social Corporativa, y aunque es cierto que la mayoría pertenecen a los primeros cursos de la carrera, también hay estudiantes de cuarto que se encuentran en esta tesitura.

Por otro lado, solamente un $12 \%$ de los encuestados reconocen que les gustaría trabajar en el sector agroalimentario gallego, mientras que un $32 \%$ afirma claramente que no tiene interés por la industria, y una mayoría (56\%) se encuentra indiferente ante este sector.

Gráfico 3: Interés que muestran los alumnos en trabajar en el sector agroalimentario gallego

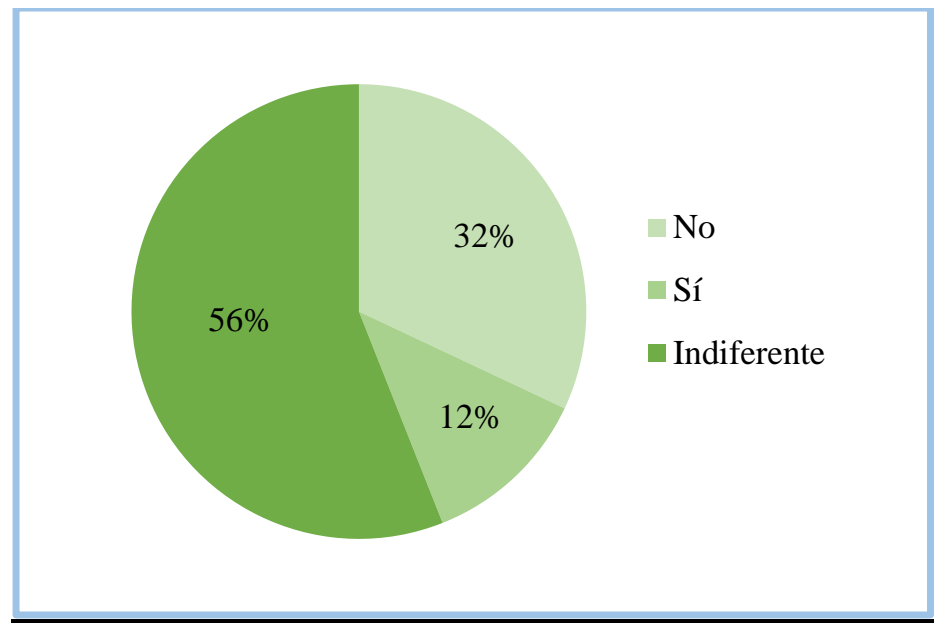

Fuente: elaboración propia

\section{Conclusiones}

El sector agroalimentario gallego es una pieza fundamental en la economía de esta comunidad. Su actividad es una de las principales tanto dentro del territorio autonómico como estatal, lo que supone que dé empleo a un gran número de personas. La conciencia que se tiene hoy en día sobre la sostenibilidad y el cuidado que hay que tener a nivel corporativo con todo lo que nos rodea ha supuesto que cada vez se valoren más este tipo de actividades por parte de las empresas y de la sociedad.

Este estudio pretendía comprobar si los estudiantes gallegos tienen en cuenta este tipo de prácticas a la hora de buscar trabajo y se ha demostrado que hoy en día, los estudiantes le darían mucha importancia a la hora de aplicar en una empresa al hecho de que ésta realizara actividades de RSC dirigidas hacia sus empleados, los clientes, los stakeholders y en menor medida, hacia el gobierno. Aunque hay que reconocer que la mayoría de los estudiantes no encuentran este sector atractivo de primeras a la hora de buscar empleo. Por lo tanto, las implicaciones que este estudio tiene son evidente. Por un lado, las universidades deberían de tener en cuenta estos datos para concienciar a los alumnos de la importancia que tiene el sector agroalimentario en Galicia y debería incidir en los conocimientos sobre RSC. Por otra parte, las empresas deberían de aprovechar esta oportunidad para realizar actividades formativas en las que comuniquen sus actividades de RSC en las Universidades y pongan en contacto a los estudiantes con este campo. Además, deberían considerar sus políticas hacia los empleados y medioambiente, principalmente, con el objetivo de atraer a un mayor número de empleados realizando RSC y fomentando un desarrollo sostenible. 


\section{Bibliografía}

Aguilera, R. V., Rupp, D. E., Williams, C. A., \& Ganapathi, J. 2007. Putting the S back in corporate social responsibility: A multilevel theory of social change in organizations. Academy of Management Review, 32: 836-863.

Conócenos | Clusaga 'Clúster Alimentario de Galicia'. (n.d.). Clúster Alimentario de Galicia. Retrieved 17 May 2021, from https://www.clusteralimentariodegalicia.org/sobre-el-clusaga

El sector agroalimentario genera en Galicia más de 103.000 puestos de trabajo, según un estudio del IGE publicado hoy—-Notas de Prensa - Consellería de Hacienda y Administración Pública. (n.d.). Retrieved 17 May 2021, from http://www.conselleriadefacenda.es/es/sala-prensa/notas-deprensa/-/publicador/TCtfwYC8Z77X/content/el-sector-agroalimentario-genera-en-galicia-masde-103-000-puestos-de-trabajo-segun-un-estudio-del-ige-publicadohoy;jsessionid=cyRfB7xJu1GEggnZ9FOV8MjS.cac6cba5-fef0-33d4-a719-d618a829dfb1

Iribarren, D., Vázquez-Rowe, I., Hospido, A., Moreira, M. T., \& Feijoo, G. (2010). Estimation of the carbon footprint of the Galician fishing activity (NW Spain). Science of The Total Environment, 408(22), 5284-5294. https://doi.org/10.1016/j.scitotenv.2010.07.082

Klimkiewicz, K., \& Oltra, V. (2017). Does CSR enhance employer attractiveness? The role of millennial job seekers' attitudes. Corporate Social Responsibility and Environmental Management, 24, 449463. https:// doi.org/10.1002/csr.1419

López-Iglesias, E. (2019). The agricultural and agri-food sector in Galicia; assessment of changes since European integration, 1986-2016. Revista Galega de Economía, 28(3), 1-20. https://doi.org/10.15304/rge.28.3.6168

Publicaciones cajamar. (n.d.). Retrieved 17 May 2021, from https://publicacionescajamar.es/

Skudiene, V. and Auruskeviciene, V. (2012), "The contribution of corporate social responsibility to internal employee motivation", Baltic Journal of Management, Vol. 7 No. 1, pp. 49-67. doi:10.1108/17465261211197421.

Turker, D. (2008). How Corporate Social Responsibility Influences Organizational Commitment. Journal of Business Ethics, 89(2), 189. https://doi.org/10.1007/s10551-008-9993-8 